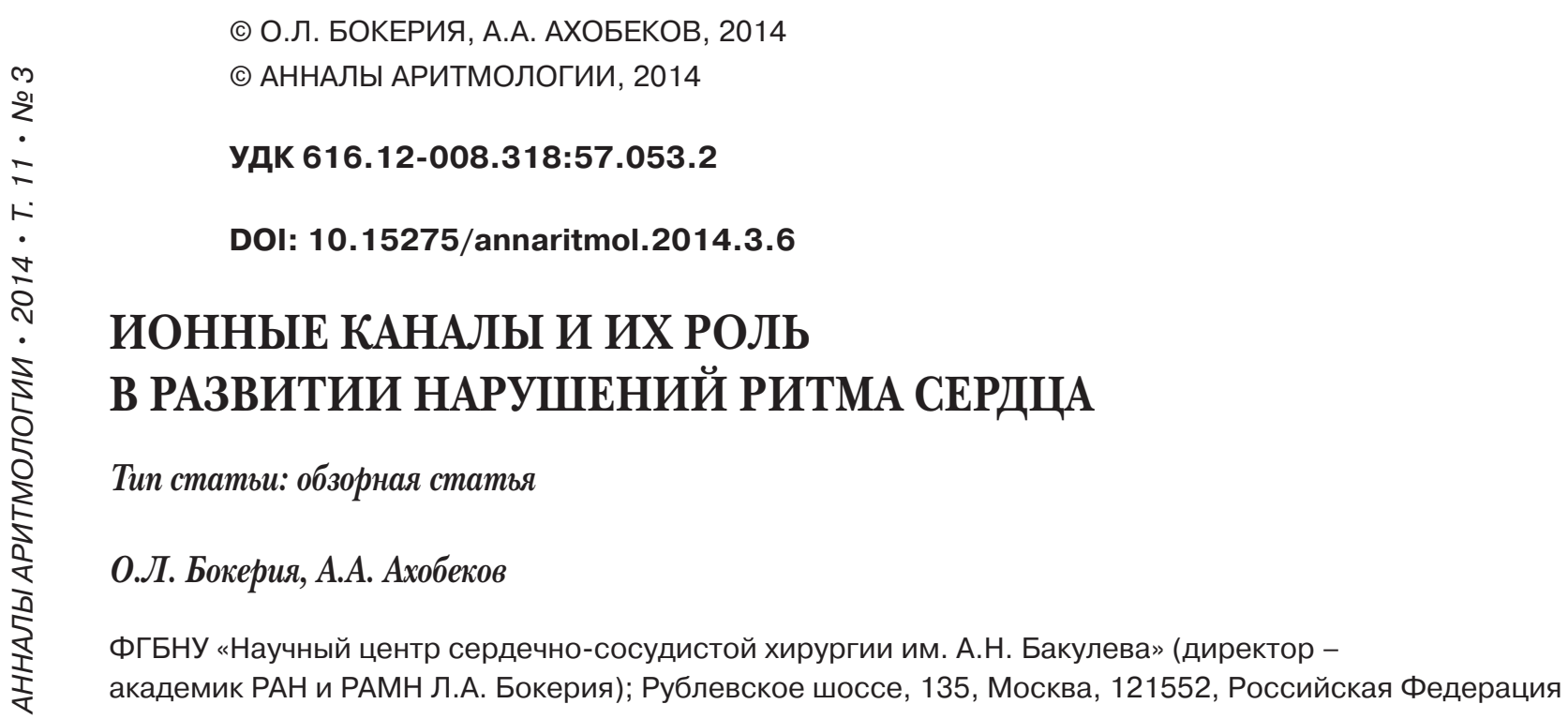


Бокерия Ольга Леонидовна, доктор мед. наук, профессор, гл. научн. сотр., зам. заведующего отделением;

Ахобеков Альберт Амалиевич, аспирант, кардиолог, e-mail: alber-t7@mail.ru

Электрическая активность сердца в физиологических условиях начинается с возбуждения клеток синоатриального (СА) узла. Медленная диастолическая деполяризация клеток СА-узла приводит к возникновению потенциала действия, который распространяется по предсердиям, атриовентрикулярному (АВ) узлу, системе Гиса-Пуркинье и миокарду желудочков, вызывая их возбуждение. Тем самым за счет электромеханического сопряжения происходит последовательное сокращение предсердий и желудочков. Потенциал действия кардиомиоцитов состоит из 5 последовательных фаз: 1) фаза быстрой деполяризации (фаза 0) начинается во время входа ионов $\mathrm{Na}^{+}$в клетку по быстрым натриевым каналам; 2) фаза ранней быстрой реполяризации (фаза 1); 3) фаза медленной деполяризации, или плато (фаза 2), когда одновременно происходит вход ионов $\mathrm{Ca}^{2+}$ по медленным кальциевым каналам и выход ионов $\mathrm{K}^{+}$; 4) фаза поздней быстрой реполяризации (фаза 3), обусловленная преобладающим выходом ионов $K^{+}$; 5) фаза 4 - потенциал покоя. Развитие и поддержание потенциала действия в миокарде регулируется работой множества белков ионных каналов. Трансмембранные белки обеспечивают постоянный ток ионов через ионные каналы к сарколеммам кардиомиоцитов, генерирующих электрические импульсы. Ионные каналы подразделяются на потенциал-, хемо- и механоуправляемые. Все ионные каналы состоят из основных $\alpha$-субъединии и дополнительных субъединии. Основной структурой ионного канала являются $\alpha$-субъединицы, которые определяют его нормальную функцию, а дополнительные $\beta$-субъединицы регулируют только кинетику канала. Правильная организация электрической активности сердиа осуществляется благодаря нормальному функционированию ионных каналов кардиомиоцитов. При мутации генов, кодирующих белки ионных каналов, развиваются так называемые каналопатии, проявляющиеся жизнеугрожающими аритмиями. Разнообразие ионных каналов сердиа и их функций предполагает комплексный подход в диагностике и лечении нарушений ритма сердиа. Понимание роли ионных токов в формировании потенциала действия в физиологических условиях и при патологических изменениях может способствовать успешному выбору антиаритмических препаратов для эффективного лечения аритмий и применению их с минимально возможной вероятностью аритмогенного действия. Во многих случаях антиаритмической терапии недостаточно для эффективной профилактики внезапной сердечной смерти на фоне жизнеугрожающих аритмий. Такой категории пациентов необходима имплантация кардиовертера-дефибриллятора. Широкое внедрение генетических и клеточных технологий в будущем, возможно, решит проблемы профилактики и лечения врожденных каналопатий сердиа.

Ключевые слова: ионные каналы; аритмии; кардиомиоциты.

\section{ION CHANNELS AND THEIR ROLE IN THE DEVELOPMENT OF ARRHYTHMIAS}

\section{O.L. Bockeria, A.A. Akhobekov}

A.N. Bakoulev Scientific Center for Cardiovascular Surgery; Rublevskoe shosse, 135, Moscow, 121552, Russian Federation

Bockeria Ol'ga Leonidovna, MD, DM, Professor, Chief Research Associate, Deputy Chief of Department; Akhobekov Al'bert Amalievich, Postgraduate, Cardiologist, e-mail: alber-t7@mail.ru

Under physiological conditions the electrical activity of the heart begins with the sinoatrial node cells excitation. The slow diastolic depolarization of sinoatrial node cells leads to an action potential that spreads through the atria, the atrioventricular node, the His-Purkinje system and ventricular myocardium, causing their excitement. Thus, due to the electromechanical coupling the sequential contraction of the atria and the ventricles occurs. The cardiac action potential consists of 5 consecutive phases: 1) rapid depolarization phase (phase 0) is due to the opening of the fast $\mathrm{Na}^{+}$channels causing a rapid influx of $\mathrm{Na}^{+}$ions into the cell; 2) the early phase of rapid repolarization (phase 1); 3) slow depolarization phase or plateau (phase 2), is sustained by a balance between inward movement of $\mathrm{Ca}^{2+}$ and outward movement of $\mathrm{K}^{+}$; 4) late phase of rapid repolarization (phase 3), due to the prevailing yield of $K^{+}$ions; 5) phase 4 - the resting membrane potential. Initiation and sustenance of the action potential in the myocardium is regulated by activity of the plurality of ion channels proteins. Transmembrane proteins provide a constant current of ions through the ion channels into the sarcolemma of cardiomyocytes, generating electrical impulses. Ion channels are divided into voltage-, ligand-and mechano-gated. All ion channels are composed of a $\alpha$-subunit core and additional subunits. The basic structure of the ion channel is $\alpha$-subunit, which determines its normal function, and $\beta$-subunits regulate only the kinetics of the channel. Proper organization of the electrical activity of the heart is carried out through the normal functioning of the cardiac ion channels. If there are mutations in genes encoding 
ion channel proteins, the so-called channelopathies develope, manifesting with life-threatening arrhythmias. Variety of ion channels of the heart and their functions assume an integrated approach to the diagnosis and treatment of cardiac arrhythmias. Understanding the role of ionic currents in the formation of an action potential under physiological conditions and pathological changes may contribute to the successful selection of antiarrhythmic drugs for the effective treatment of arrhythmias and their using with the least possible chance of proarrhythmogenic action. In many cases the only antiarrhythmic therapy is insufficient for effective prevention of sudden cardiac death in consequence of the life-threatening arrhythmias. This category of patients requires implantation of a cardioverter-defibrillator. In the future the widespread application of genetic and cellular technologies may solve the problem of prevention and treatment of congenital cardiac channelopathies.

Key words: ion channels; arrhythmias; cardiomyocytes.

\section{Введение}

Э лектрическая активность сердца в физиологических условиях начинается с возбуждения клеток синоатриального (СА) узла. Медленная диастолическая деполяризация клеток СА-узла приводит к возникновению потенциала действия, который распространяется по предсердиям, атриовентрикулярному (АВ) узлу, системе Гиса-Пуркинье и миокарду желудочков, вызывая их возбуждение. Тем самым за счет электромеханического сопряжения происходит последовательное сокращение предсердий и желудочков. Медленная диастолическая деполяризация характерна как для клеток СА-узла, так и для клеток АВ-узла, пучка Гиса и волокон Пуркинье, однако это свойство проявляется только при отказе СА-узла или его слабости, так как в нормальных условиях их спонтанная активность подавляется более частыми импульсами от СА-узла (overdrive suppression). Медленная диастолическая деполяризация не характерна для клеток рабочего миокарда из-за того, что в покое внутриклеточный потенциал клеток имеет отрицательное значение, что обусловлено большей проницаемостью клеточной мембраны для ионов $\mathrm{K}^{+}[1]$.

Потенциал действия кардиомиоцитов состо₹ $\quad$ ит из 5 последовательных фаз: 1) фаза быстрой деполяризации (фаза 0), начинается во время входа ионов $\mathrm{Na}^{+}$в клетку по быстрым натриевым каналам; 2) фаза ранней быстрой реполяризации (фаза 1); 3) фаза медленной деполяризации, или плато (фаза 2), когда одновременно происходит вход ионов $\mathrm{Ca}^{2+}$ по медленным кальциевым каналам и выход ионов $\mathrm{K}^{+}$; 4) фаза поздней быстрой реполяризации (фаза 3), обусловленная преобладающим выходом ионов $\mathrm{K}^{+}$; 5) фаза 4 - потенциал покоя.

Развитие и поддержание потенциала действия в миокарде регулируется работой множества белков ионных каналов (см. рисунок). Трансмембранные белки обеспечивают постоянный ток ионов через ионные каналы к сарколеммам кардиомиоцитов, генерирующих электрические импульсы. Каждый ионный канал представляет собой белковую молекулу, расположенную в мембране кардиомиоцита. Все ионные каналы в разное время находятся в одном из трех состояний: покоя, активации и инактивации. Благодаря переходу ионных каналов из состояния покоя в состояние активации происходит ток ионов, формирующий мембранный ток. В мембране каждого кардиомиоцита располагаются около 100 тыс. ионных каналов. Большинство каналов являются селективными и пропускают только один вид ионов. Ионные каналы подразделяются на потенциал-, хемо- и механоуправляемые [2]. Все ионные каналы состоят из основных $\alpha$-субъединиц и дополнительных субъединиц. Основной структурой ионного канала являются $\alpha$-субъединицы, которые определяют его нормальную функцию, а дополнительные $\beta$-субъединицы регулируют кинетику канала. Правильная организация электрической активности сердца осуществляется благодаря нормальному функционированию ионных каналов кардиомиоцитов. При мутации генов, кодирующих белки ионных каналов, развиваются так называемые каналопатии, проявляющиеся жизнеугрожающими аритмиями. В данной статье будут рассмотрены современные данные о нормальной физиологии и патологии ионных каналов кардиомиоцитов, а также сопровождающих их жизнеугрожающих аритмиях.

\section{Входящие ионные токи}

\section{Быстрый натриевый ток (INa)}

INa осуществляется через потенциалуправляемые $\mathrm{Na}^{+}$-каналы и обеспечивает быструю деполяризацию (0 фаза) в кардиомиоцитах желудочков, предсердий и в клетках волокон Пуркинье. Потенциалуправляемый $\mathrm{Na}^{+}$-канал состоит из одной $\alpha$-субъединицы и двух $\beta$-субъединиц. 


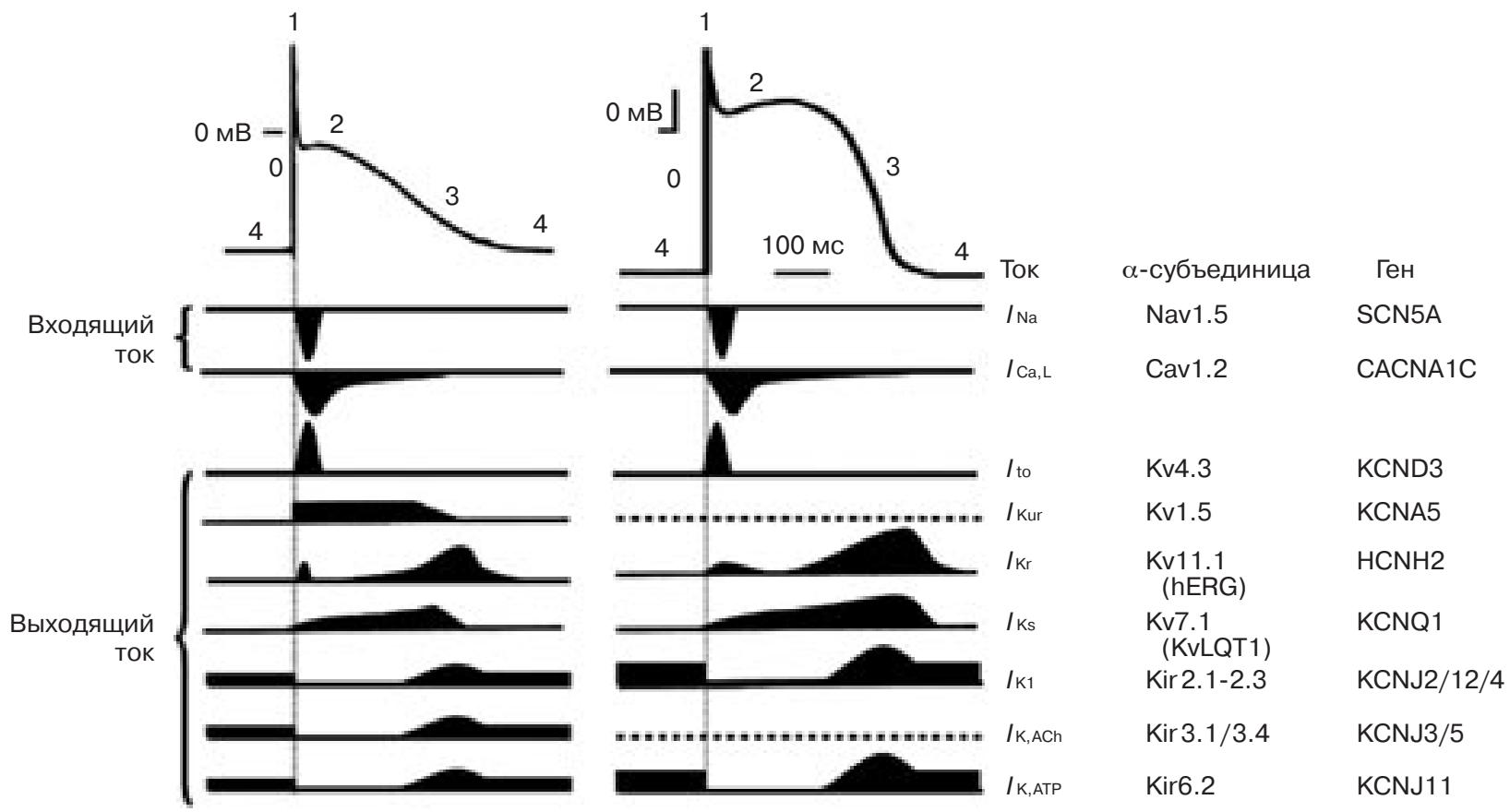

Натриевые, калиевые, кальциевые ионные каналы

Основные функции канала (чувствительность к уровню мембранного потенциала, активация и инактивация) связаны с $\alpha$-субъединицей. В настоящее время известно только одно подсемейство $\alpha$-субъединиц Na-каналов - Nav1, в котором выделяют 9 изоформ. Из них Nav1.5 является единственной субъединицей, экспрессированной в сердце человека. При нормальных физиологических условиях в течение фазы плато потенциала действия кардиомиоцита около 99\% каналов Nav инактивированы. Нарушение инактивации Nav приводит к развитию жизнеугрожающих аритмий сердца [3].

Основная $\alpha$-субъединица натриевого канала, формирующего натриевый ток Nav1.5, кодируется геном SCN5A. Мутация этого гена связана с развитием таких тяжелых аритмий сердца, как врожденный синдром удлиненного интервала $Q-T$ (LQTS 3), синдром детской внезапной смерти, идиопатическая фибрилляция желудочков, синдром слабости синусного узла (2-й тип), синдром Бругада (1-й тип), аритмогенная дисплазия правого желудочка (5-й тип) и прогрессирующее нарушение проводимости, болезнь Лева-Ленегра.

Так, мутация в гене SCN5A, связанная с развитием врожденного синдрома удлиненного интервала $Q-T$ (LQTS 3), вызывает дефект инактивации натриевых каналов, приводящий к появлению позднего натриевого тока, отсутст- вующего в норме, который нейтрализует выходящий $\mathrm{K}^{+}$ток и удлиняет «плато», что приводит к удлинению потенциала действия желудочков (удлинение интервала $Q-T$ ). В свою очередь удлинение интервала $Q-T$ повышает вероятность возникновения ранних постдеполяризаций, вызывающих полиморфную желудочковую тахикардию по типу «torsade de point's» $[4,5]$.

В отличие от LQTS 3, синдром Бругада характеризуется замедлением деполяризующего натриевого тока и, как следствие, замедлением скорости быстрой деполяризации в эпикарде правого желудочка. Снижение плотности $\mathrm{Na}^{+}$-каналов по отношению к плотности кратковременного выходящего тока Ito приводит к преждевременной реполяризации и укорочению потенциала действия в эпикарде, тогда как в эндокарде деполяризация внутренних слоев мембраны протекает нормально. В этой связи развивается электрическая гетерогенность миокарда правого желудочка. Как следствие, деполяризованный эндокард может стать источником повторного возбуждения преждевременно реполяризованного эпикарда, и возможно развитие полиморфной желудочковой тахикардии (ЖТ) [6, 7].

Синдром Лева-Ленегра представлен прогрессирующим замедлением проведения по всей проводящей системе сердца без увеличения риска развития жизнеугрожающих желудочковых аритмий. При синдроме Лева-Ленегра для ак- 
тивации $\mathrm{Na}^{+}$-канала требуется воздействие сверхпорогового стимула [8]. Морфологическим субстратом данного синдрома является прогрессирующее склеродегенеративное поражение проводящей системы сердца. На ЭКГ данный синдром может быть представлен сочетанием полной блокады правой ножки пучка Гиса и блокады передневерхнего разветвления левой ножки пучка Гиса [9].

Врожденный синдром удлиненного интервала $Q-T$ (LQTS4) является следствием мутации в гене, кодирующем анкирин В. Анкирин В входит в группу многофункциональных адаптерных мембранных белков, участвующих в регуляции работы $\mathrm{Na}^{+}$-канала. LQTS 4 является наименее изученным из всех типов LQTS [10].

\section{Кальциевые токи (ICa, L и ICa, T)}

В кардиомиоцитах обнаружены два потенциалуправляемых типа $\mathrm{Ca}^{2+}$-токов: L- и T-типа. $\mathrm{Ca}^{2+}$-каналы клеток сердца состоят из основной субъединицы $\alpha 1$ и вспомогательных субъединиц $\alpha 2 d$ и $\beta 2$. ICa L-типа больше представлен в кардиомиоцитах желудочков по сравнению с кардиомиоцитами предсердий. В клетках СА- и АВ-узла ICa L-типа формируют спонтанную деполяризацию и проведение импульса.

Данные каналы сформированы из $\alpha 1$-субъединиц семейства Cav1.x. Из них в сердце встречаются изоформы Cav1.2 ( $\alpha 1 \mathrm{C})$ и Cav1.3 ( $\alpha 1 \mathrm{D})$. ICa L-типа ингибируются такими антагонистами кальция, как верапамил, нифедипин, дилтиазем [11].

ICa T-типа в наибольшей стапени представлены в пейсмейкерных клетках и проводящей системе сердца. Амплитуда тока ICa Т-типа составляет $20 \%$ от амплитуды тока ICa L-типа. Они состоят из $\alpha 1$-субъединиц семейства ₹ Cav3.x, в сердце встречаются изоформы Cav3.1 $(\mathrm{a} 1 \mathrm{G})$ и Cav3.2 $(\alpha 1 \mathrm{H})$. В отличие от ICa L-типа, они не чувствительны к внутриклеточной концентрации $\mathrm{Ca}^{2+}$ и не блокируются классическими антагонистами кальциевых каналов.

Существенная роль в поддержании внутриклеточного гомеостаза ионов $\mathrm{Ca}^{2+}$ принадлежит рианодиновым рецепторам RyR2. Нарушение их функции приводит к избыточному выходу $\mathrm{Ca}^{2+}$ из саркоплазматического ретикулума, вызывая перегрузку $\mathrm{Ca}^{2+}$ и повышая триггерную активность миокарда желудочков.

Рианодиновый рецептор является основной структурой кальциевых каналов в саркоплазматическом ретикулуме кардиомиоцитов [8]. Ос- новополагающая роль рианодиновых рецепторов заключается в активизации потенциалзависимых кальциевых каналов в плазмалемме. N. Liu и соавт. впервые выявили, что дисфункция рианодиновых рецепторов (RyR2) является причиной развития катехоламинергической полиморфной желудочковой тахикардии. Результатом мутаций в гене RyR2 является развитие около 55\% случаев катехоламинергической полиморфной желудочковой тахикардии [12].

Второй генотип катехоламинергической полиморфной желудочковой тахикардии (CVPT2) связан с мутациями в гене кальсеквестрина-2 (CASQ2), картированного на 1-й хромосоме в локусе 1p13.3-p11. Кальсеквестрин-2 является основным кальцийсвязывающим белком в саркоплазматическом ретикулуме кардиомиоцитов.

Белки RyR2 и CASQ2 вовлечены в один внутриклеточный метаболический процесс, связанный с контролем потоков внутриклеточного кальция и концентрации свободного кальция в цитоплазме. Вследствие мутаций в обоих генах происходит усиленное высвобождение ионов кальция из саркоплазматического ретикулума в ответ на вход ионов кальция в клетку, вызывая перегрузку клеток данными ионами, что усиливает трансмембранную дисперсию реполяризации и запускает ЖТ по механизму риентри [11].

\section{Пейсмейкерный ток (If)}

If - это входящий неселективный $\mathrm{Na}^{+}$- и $\mathrm{K}^{+}$ток, который активизируется при гиперполяризации мембран пейсмейкерных клеток. If в пейсмейкерных клетках СА-узла возникает во время фазы реполяризации потенциала действия и вносит большой вклад в медленную диастолическую деполяризацию (МДД) пейсмейкерных клеток. If проходит через каналы, сформированные субъединицами семейства HCN. В настоящее время известны 4 изоформы $\mathrm{HCN}$, HCN1-HCN4, из которых в сердце экспрессируются HCN1, HCN2 и НCN4. В СА-узле и системе Пуркинье в большей степени выражена экспрессия HCN4 и в меньшей - HCN1 и HCN2. Действие ацетилхолина и агонистов бета-адренорецепторов на If происходит через изменение активности фермента аденилатциклазы и выработку циклического аденозинмонофосфата (цАМФ). Влияние цАМФ на If обусловлено прямым взаимодействием с каналом, через который этот ток протекает. Снижение функции If каналов может стать причиной развития синдрома слабости синусного узла [9]. 


\section{Выходящие токи}

\section{Кратковременный выходящий ток (Ito)}

Ito участвует в фазе ранней реполяризации потенциала действия кардиомиоцитов и клеток проводящей системы Гиса-Пуркинье. Ito состоит из потенциалчувствительного, $\mathrm{Ca}^{2+}$-независимого $\mathrm{K}^{+}$-тока Ito1 и активируемого кальцием Cl-тока Ito2.

Ito1 обнаружен в предсердиях и желудочках многих видов животных, а также в СА-узле кролика. Этот селективный $\mathrm{K}^{+}$-ток быстро активируется и инактивируется, он не чувствителен к $\mathrm{Ca}^{2+}$, блокируется 4-аминопиридином.

Ito2 участвует в фазе ранней реполяризации вместе с Ito1. При перегрузке $\mathrm{Ca}^{2+}$ Ito2 может вносить вклад в аритмогенный ток Iti, участвующий в триггерной активности. Ito2 подавляется ингибиторами ионного транспорта DIDS, SITS и нифлюмовой кислотой [13].

\section{Ток задержанного выпрямления (IK)}

IK называется таким образом в связи с относительно медленной активацией по сравнению с другими токами. Известны три компонента IK - IKs, IKr и IKur, которые различаются по временной кинетике и фармакологической чувствительности [14].

IKs (медленный IK) активируется достаточно медленно (за 800 мс при 0 мВ), блокируется хроманолом 293B и L-735821. IKs протекает через каналы проводимостью 4,5 пСм, которые образованы из субъединицы KvLQT1 и дополнительной $\operatorname{minK}$.

$\mathrm{IKr}$ (быстрый IK) активируется относительно быстро (за 200 мс при потенциале 0 мВ). IKr блокируется антиаритмическими препаратами III класса. Проводимость каналов, через которые протекает IKr, равна 2 пСм. Молекула канала состоит из основной субъединицы HERG и дополнительной MiRP1.

IKur (сверхбыстрый IK) очень быстро активируется (за 10 мс при 0 мВ) и очень быстро инактивируется. Каналы, через которые протекает ток IKur, образованы из субъединиц Kv1.5.

Bce три компонента IK играют основную роль в реполяризации в конце фаз 2 и 3 потенциала действия кардиомиоцитов. Стоит отметить, что из-за разной скорости инактивации их вклад в реполяризацию напрямую зависит от частоты сердечных сокращений. Увеличение IKs при повышении частоты сердечных сокращений приводит к ускорению реполяризации и, следо- вательно, укорочению потенциала действия (так называемая «частотная адаптация»). Уменьшение длительности потенциала действия приводит к снижению степени активации IKr. Вместе с увеличением амплитуды IKs это обеспечивает повышение роли IKs в реполяризации кардиомиоцитов. Такое изменение токов лежит в основе частотной зависимости действия селективных блокаторов $\mathrm{K}^{+}$-каналов. IKr и IKs из-за их медленной инактивации могут играть важную роль в формировании МДД пейсмейкерных клеток СА-узла. Различающаяся продолжительность потенциала действия в кардиомиоцитах разных отделов сердца обусловлена неодинаковым уровнем экспрессии каналов, через которые протекают IKur, IKr и IKs. Более короткий потенциал действия в предсердиях связан с наличием в них в большом количестве IKur, который отсутствует в желудочках. А также амплитуда $\mathrm{IKr}$ намного больше в левом, чем в правом предсердии, в результате чего потенциал действия в левом предсердии относительно короче. В желудочках $\mathrm{IKr}$ выражен в большей степени в кардиомиоцитах эпикарда и верхушки, где потенциал действия также короче. Достаточно большая длительность потенциала действия в желудочковых М-клетках является следствием низкой амплитуды IKs. Важная роль IK в реполяризации клеток сердца подтверждается рядом врожденных и приобретенных форм синдрома удлиненного интервала $Q-T$, которые обусловлены нарушением функций этих каналов.

Так, две мутации гена, кодирующего $\alpha$-субъединицу IKs, являются причинами двух врожденных синдромов: LQTS1 и LQTS-JLN1 (1-я форма синдрома Jervell и Lange-Nielsen). А две мутации гена $\beta$-субъединицы (MinK) IKs обусловливают синдромы LQTS5 и LQT-JLN2 [14].

Мутация $\alpha$-субъединицы IKr (HERG) является причиной развития синдрома LQTS2, а мутация $\beta$-субъединицы (MiRP1) этого же канала приводит к синдрому LQTS6 [15].

Bсе перечисленные синдромы объединяет удлинение интервала $Q-T$, которое становится субстратом для развития жизнеугрожающих желудочковых тахикардий.

Укорочение интервала $Q-T$ вызывает мутации в генах калиевых каналов, приводящие к повышению активности IKr, укорочению длительности фазы реполяризации потенциала действия и уменьшению продолжительности рефрактерного периода миокарда желудочков, что сопровождается уменьшением длины волны 
возбуждения, предрасполагающее к возникновению желудочковых аритмий по механизму риентри.

\section{Ток аномального (входящего) выпрямления (IK1)}

IK1 располагается в волокнах Пуркинье, кардиомиоцитах предсердий и желудочков. IK1 получил название аномального (входящего) выпрямления из-за того, что каналы, обеспечивающие его ток, активируются только при отрицательных потенциалах и чаще проводят входящий ток, чем выходящий. IK1 участвует в реполяризации и поддержании потенциала покоя клеток. При положительных потенциалах IK1 инактивируется.

Мутации в гене, кодирующем канал IK1 (Kir2.1), приводят к развитию LQTS7 (синдром Андерсена-Тавила) [11, 16]. Нарушение функции белка Kir2.1 приводит к удлинению 3-й фазы реполяризации, соответственно удлиняется и потенциал действия кардиомиоцитов. В свою очередь удлинение потенциала действия вызывает кальциевую перегрузку, активирует входящий натрий-кальциевый обменный ток ( $\mathrm{INa} / \mathrm{Ca})$, что вызывает раннюю деполяризацию 3-й фазы и удлиняет следовую деполяризацию 4-й фазы, приводя к желудочковой тахикардии [17].

\section{Аиетилхолинактивируемый $K^{+}-$ток IK (ACh)}

IK (ACh) больше всего встречается в пейсмейкерных и предсердных клетках и волокнах Пуркинье. Активация каналов, через которые протекает IK (ACh), происходит за счет действия ацетилхолина на М2-холинорецепторы кардиомиоцитов. Активация IK (ACh) вызывает гиперполяризацию и значительное укорочение длительности потенциала действия пейсмейкерных и предсердных клеток. Кроме того, активация IK (ACh) играет важную роль в отрицательном хронотропном действии ацетилхолина. IK (ACh) является потенциалчувствительным, для него характерно аномальное (входящее) выпрямление, за счет чего он играет основную роль при потенциалах, близких к потенциалу покоя, и практически отсутствует во время фазы деполяризации и плато потенциала действия [18].

\section{АТФ-чувствительный $\mathrm{K}^{+}$-ток IК (АТФ)}

IK (АТФ) обусловливает связь между метаболизмом сердца и его электрической активностью. IK (АТФ) подавляется внутриклеточным
АТФ. Точнее, внеклеточный АТФ ингибирует IK (АТФ) в микромолярных концентрациях, а внутриклеточный АТФ - в миллимолярных концентрациях. Следовательно, при физиологических условиях IK (АТФ) инактивирован. Если внутриклеточная концентрация АТФ снижается из-за ишемии или гипоксии, IK (АТФ) активируется, приводя к укорочению потенциала действия и развитию жизнеугрожающих желудочковых аритмий сердца [19].

\section{Механозависимые ионные токи}

Известно, что электрофизиологические параметры сердца меняются при его растяжении. Такие изменения связаны с ионными токами, протекающими через специализированные ионные каналы, чувствительные к растяжению (stretch-activated channels (SAC)). Большинство SAC являются неселективными, то есть они пропускают в равной степени ионы $\mathrm{Ca}^{2+}, \mathrm{Na}^{+}$ и $\mathrm{K}^{+}$, в то время как другие каналы селективны для определенных ионов. Наличие входящих и выходящих токов (ISAC), протекающих через $\mathrm{SAC}$, объясняет различные механизмы влияния растяжения на электрофизиологические параметры сердца. Так, активация выходящих ISAC ускоряет реполяризацию во время фазы плато, что приводит к укорочению длительности потенциала действия и, как следствие, эффективного рефрактерного периода. Наиболее изучено молекулярное строение $\mathrm{K}^{+} \mathrm{SAC}$, которые состоят из субъединиц TREK-1. Эти каналы активируются при механическом растяжении, а также при действии арахидоновой кислоты.

\section{Ионные насосы}

В кардиомиоцитах и пейсмейкерных клетках, кроме ионных каналов, особую роль в формировании потенциала действия играют ионные насосы и обменники. Они обеспечивают поддержание постоянного внутриклеточного уровня ионов $\mathrm{K}^{+}, \mathrm{Na}^{+}$и $\mathrm{Ca}^{2+}$. INa/K-насос (INa/K pump) обеспечивает активный транспорт ионов $\mathrm{Na}^{+}$и $\mathrm{K}^{+}$через клеточную мембрану. INa/K-насос относится к АТФ-азам Р-типа и состоит из $\alpha$ - и $\beta$-субъединиц. Выявлены 4 изоформы $\alpha$ - и $\beta$-субъединиц, которые в любом сочетании дают работающий насос. INa/K-насос может приводить к гиперполяризации, укорочению длительности потенциала действия и замедлению спонтанной активности. Работа INa/K-наcoca зависит от внутриклеточной концентра- 
ции $\mathrm{Na}^{+}$. Работа INa/K-насоса подавляется сердечными гликозидами, в частности дигиталисом.

Ток Na/Ca-обмена формируется за счет работы электрогенного $\mathrm{Na}^{+} / \mathrm{Ca}^{2+}$-обменного механизма, за один цикл работы которого происходит поступление внутрь клетки через клеточную мембрану 3 ионов $\mathrm{Na}^{+}$на 1 ион $\mathrm{Ca}^{2+}$. Направление INa/Cа зависит от соотношения между $\mathrm{Na}^{+}-$ и $\mathrm{Ca}^{2+}$-градиентами и мембранным потенциалом. Во время 4-й фазы потенциала действия $\mathrm{Na} / \mathrm{Ca-обмен} \mathrm{генерирует} \mathrm{небольшой} \mathrm{входящий}$ ток. После быстрой деполяризации (фаза 0) INa/Ca может на короткое время быть выходящим, затем, когда внутриклеточная концентрация $\mathrm{Ca}^{2+}$ увеличивается, он становится входящим. Таким образом, INa/Ca участвует в формировании плато (фаза 2) потенциала действия в кардиомиоцитах и обеспечивает выход ионов $\mathrm{Ca}^{2+}$ из клеток. Подавление INa/Ca приводит к укорочению длительности потенциала действия. INa/Ca может также играть важную роль в формировании МДД в пейсмейкерных клетках.

Работа $\mathrm{Na}^{+} / \mathrm{Ca}^{2+}$-обменника регулируется $\mathrm{pH}, \quad \alpha$-адренергической стимуляцией. Роль

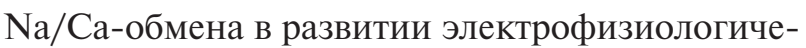
ских нарушений кардиомиоцитов хорошо представлена при ишемии и реперфузии миокарда. Ацидоз, возникающий при ишемии, приводит

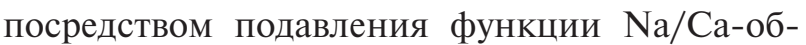
мена к повышению внутриклеточной концентрации $\mathrm{Na}^{+}$. Во время реперфузии происходит быстрое восстановление $\mathrm{pH}$ клеток снаружи и появляется градиент рН, который компенсируется за счет $\mathrm{Na}^{+} / \mathrm{H}^{+}$-обмена. При этом повышается внутриклеточная концентрация $\mathrm{Na}^{+}$,

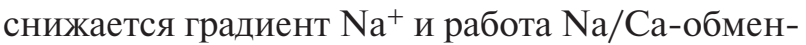
ного механизма, что приводит к перегрузке кардиомиоцитов ионами $\mathrm{Ca}^{2+}$. Перегрузка кардиомиоцитов ионами $\mathrm{Ca}^{2+}$ предрасполагает к возникновению поздних постдеполяризаций и, следовательно, желудочковых аритмий. Таким образом, подавление обратной работы $\mathrm{Na} / \mathrm{Ca}-$ обменного механизма является мишенью для профилактики и лечения реперфузионных аритмий. Примерно такие же нарушения происходят и при сердечной недостаточности. Так, при сердечной недостаточности повышается уровень

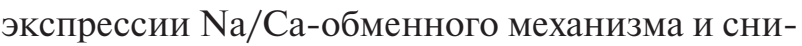
жается уровень $\mathrm{Ca}^{2+}$ АТФ-азы в саркоплазматическом ретикулуме. Снижение уровня $\mathrm{Ca}^{2+}$ АТФ-азы в саркоплазматическом ретикулуме приводит к перегрузке кардиомиоцитов ионами $\mathrm{Ca}^{2+}$ с последующим развитием желудочковых аритмий.

\section{Заключение}

Генетически обусловленные нарушения функционирования ионных каналов или регуляторных белков могут приводить к развитию сложных нарушений ритма сердца. Разнообразие ионных каналов сердца и их функций предполагает комплексный подход в диагностике и лечении нарушений ритма сердца. Понимание роли ионных токов в формировании потенциала действия в физиологических условиях и при патологических изменениях может способствовать успешному выбору антиаритмических препаратов для эффективного лечения аритмий и применению их с минимально возможной вероятностью аритмогенного действия. Во многих случаях антиаритмической терапии недостаточно для эффективной профилактики внезапной сердечной смерти на фоне жизнеугрожающих аритмий. Такой категории пациентов необходима имплантация кардиовертера-дефибриллятора. Широкое внедрение генетических и клеточных технологий в будущем, возможно, решит проблемы профилактики и лечения врожденных каналопатий сердца.

\section{Конфликт интересов}

Конфликт интересов не заявляется.

\section{Библиографический список}

1. Mangoni M., Nargeot J. Genesis and regulation of the heart automaticity. Physiol. Rev. 2005; 88 (2): 919-82. DOI: 10.1152/physrev.00018.2007.

2. Meregalli P., Wilde A., Tan H. Pathophysiological mechanism of Brugada syndrome: depolarization disorder, repolarization disorder, or more? Cardiovasc. Res. 2005; 67: 367-78. DOI: 10.1016/j.cardiores.2005.03.005

3. Ueda K., Valdivia C., Medeiros-Domingo A. et al. Syntrophin mutation associated with long QT syndrome through activation of the nNOS-SCN5A macromolecular complex. Proc. Natl. Acad. Sci. USA. 2008; 105: 9355-60 DOI: 10.1073/ pnas.0801294105.

4. Morita H., Wu J., Zipes D. The QT syndromes: long and short Lancet. 2008; 372: 750-63. DOI: 10.1016/S0140-6736(08) 61307-0.

5. Шомахов Р.А., Макаренко В.Н., Бокерия Л.А. Эволюция методов диагностики и прогнозирования некоронарогенных желудочковых аритмий. Креативная кардиология. 2014; 2: 36-47.

6. Watanabe H., Koopmann T, Le Scouarnec S, et al. Sodium channel $\alpha 1$ subunit mutations associated with Brugada syndrome and cardiac conduction disease in humans. J. Clin. Invest. 2008; 118: 2260-68. DOI: 10.1172/JCI33891.

7. Hu D., Barajas-Martinez H., Burashnikov E. et al. A mutation in the $\alpha 3$ subunit of the cardiac sodium channel associated with Brugada ECG phenotype. Circ. Cardiovasc. Genet. 2009; 2: 270-8. DOI: 10.1161/CIRCGENETICS.108.829192. 
8. Grant A., Carboni M., Neplioueva V. et al. Long QT syndrome, Brugada syndrome, and conduction system disease are linked to a single sodium channel mutation. J. Clin. Invest. 2002; 110: 1201-9. DOI: 10.1172/JCI15570.

9. Бокерия Л.А., Бокерия О.Л., Кудзоева З.Ф. Прогрессирующее замедление проводимости (болезнь Лева-Ленегра). Анналы аритмологии. 2010; 7: 26-35.

10. Verkerk A., Wilders R., van Borren M. et al. Pacemaker current (If) in the human sinoatrial node. Eur. Heart J. 2007; 28: 2472-8. DOI: 10.1093/eurheartj/ehm339.

11. Splawski I., Timothy K., Decher N. et al. Severe arrhythmia disorder caused by cardiac L-type calcium channel mutations. Proc. Natl. Acad. Sci. USA. 2005; 102: 8089-96. DOI: 10.1073/pnas.0502506102

12. Liu N., Ruan Y., Priori S. Catecholaminergic polymorphic ventricular tachycardia. Prog. Cardiovasc. Dis. 2008; 51: 23-30. DOI: $10.1016 /$ j.pcad.2007.10.005.

13. Oudit G., Kassiri Z., Sah R. et al. The molecular physiology of the cardiac transient outward potassium current (I(to)) in normal and diseased myocardium. J. Mol. Cell. Cardiol. 2001; 33 (5): 851-72. DOI: 10.1006/jmcc.2001.1376.

14. Howarth F., Hancox J. Characteristics of the delayed rectifier K current compared in myocytes isolated from the atrioventricular node and ventricle of the rabbit heart. Eur. J. Electrophysiol. 1996; 431 (5): 713-22.

15. Amin A., Herfst L., Delisle B. et al. Fever-induced QTc prolongation and ventricular arrhythmias in individuals with type 2 congenital long QT syndrome. J. Clin. Invest. 2008; 118 2552-61. DOI: 10.1172/JCI35337.

16. Tristani-Firouzi M., Etheridge S. Kir 2.1 channelopathies: the Andersen-Tawil syndrome. Eur. J. Physiol. 2010. 460: 289-94. DOI: $10.1007 / \mathrm{s} 00424-010-0820-6$.

17. Levine E., Rosero S., Budzikowski A., Moss A. et al. Congenita long QT syndrome: considerations for primary care physicians. Cleve. Clin. J. Med. 2009; 75 (8): 591-600.

18. Sakmann B., Noma A., Trautwein W. Acetylcholine activation of single muscarinic $\mathrm{K}^{+}$channels in isolated pacemaker cells of the mammalian heart. Nature. 1983; 250-3.

19. Racay T., Gregory P., Schwaller B. et al. Preconditioning experiments showed that protecting mitochondrial integrity is highly associated with cell survival following ischemia. Heart. 2006; 92; 619-24.

\section{References}

1. Mangoni M., Nargeot J. Genesis and regulation of the heart automaticity. Physiol. Rev. 2005; 88 (2): 919-82. DOI: 10.1152/physrev.00018.2007.

2. Meregalli P., Wilde A., Tan H. Pathophysiological mechanisms of Brugada syndrome: depolarization disorder, repolarization disorder, or more? Cardiovasc. Res. 2005; 67: 367-78. DOI: 10.1016/j.cardiores.2005.03.005

3. Ueda K., Valdivia C., Medeiros-Domingo A. et al. Syntrophin mutation associated with long QT syndrome through activation of the nNOS-SCN5A macromolecular complex. Proc.
4. Morita H., Wu J., Zipes D. The QT syndromes: long and short. Lancet. 2008; 372: 750-63. DOI: 10.1016/S0140-6736(08) 61307-0.

5. Shomakhov R.A., Makarenko V.N., Bockeria L.A. Evolution of diagnostic methods and prediction of noncoronary ventricular arrhythmias. Kreativnaya Kardiologiya. 2014; 2: 36-47 (in Russian).

6. Watanabe H., Koopmann T., Le Scouarnec S. et al. Sodium channel $\beta 1$ subunit mutations associated with Brugada syndrome and cardiac conduction disease in humans. J. Clin. Invest. 2008; 118: 2260-68. DOI: 10.1172/JCI33891.

7. Hu D., Barajas-Martinez H., Burashnikov E. et al. A mutation in the $\beta 3$ subunit of the cardiac sodium channel associated with Brugada ECG phenotype. Circ. Cardiovasc. Genet. 2009; 2: 270-8. DOI: 10.1161/CIRCGENETICS.108.829192.

8. Grant A., Carboni M., Neplioueva V. et al. Long QT syndrome, Brugada syndrome, and conduction system disease are linked to a single sodium channel mutation. J. Clin. Invest. 2002; 110: 1201-9. DOI: 10.1172/JCI15570.

9. Bockeria L.A., Bockeria O.L., Kudzoeva Z.F. Progressive deceleration of conductivity (Lev-Lenegr disease). Annaly Aritmologii. 2010; 7: 26-35 (in Russian).

10. Verkerk A., Wilders R., van Borren M. et al. Pacemaker current (If) in the human sinoatrial node. Eur. Heart J. 2007; 28: 2472-8. DOI: 10.1093/eurheartj/ehm339.

11. Splawski I., Timothy K., Decher N. et al. Severe arrhythmia disorder caused by cardiac L-type calcium channel mutations. Proc. Natl. Acad. Sci. USA. 2005; 102: 8089-96. DOI: 10.1073/pnas.0502506102.

12. Liu N., Ruan Y., Priori S. Catecholaminergic polymorphic ventricular tachycardia. Prog. Cardiovasc. Dis. 2008; 51: 23-30. DOI: 10.1016/j.pcad.2007.10.005.

13. Oudit G., Kassiri Z., Sah R. et al. The molecular physiology of the cardiac transient outward potassium current (I(to)) in normal and diseased myocardium. J. Mol. Cell. Cardiol. 2001; 33 (5): 851-72. DOI: 10.1006/jmcc.2001.1376.

14. Howarth F., Hancox J. Characteristics of the delayed rectifier K current compared in myocytes isolated from the atrioventricular node and ventricle of the rabbit heart. Eur. J. Electrophysiol. 1996; 431 (5): 713-22.

15. Amin A., Herfst L., Delisle B. et al. Fever-induced QTc prolongation and ventricular arrhythmias in individuals with type 2 congenital long QT syndrome. J. Clin. Invest. 2008; 118: 2552-61. DOI: 10.1172/JCI35337.

16. Tristani-Firouzi M., Etheridge S. Kir 2.1 channelopathies: the Andersen-Tawil syndrome. Eur. J. Physiol. 2010. 460: 289-94. DOI: $10.1007 / \mathrm{s} 00424-010-0820-6$.

17. Levine E., Rosero S., Budzikowski A., Moss A et al. Congenital long QT syndrome: considerations for primary care physicians. Cleve. Clin. J. Med. 2009; 75 (8): 591-600.

18. Sakmann B., Noma A., Trautwein W. Acetylcholine activation of single muscarinic $\mathrm{K}^{+}$channels in isolated pacemaker cells of the mammalian heart. Nature. 1983; 250-3.

19. Racay T., Gregory P., Schwaller B. et al. Preconditioning experiments showed that protecting mitochondrial integrity is highly associated with cell survival following ischemia. Heart. 2006; 92; 619-24.

Поступила 21.11.2014 г Подписана в печать 28.11.2014 г. 